\title{
Turbo-Codes and Turbo-Coded Modulation in CDMA Mobile Radio Systems for Short Frame Transmission
}

\author{
Volker Kühn \\ University of Bremen (Germany) \\ Department of Telecommunications \\ Kufsteiner Straße NW 1 \\ D-28359 Bremen \\ e-mail: kuehn@comm.uni-bremen.de
}

\begin{abstract}
Recently, binary parallel concatenated recursive convolutional codes termed Turbo-Codes received great attention due to their amazing error correcting capabilities. Nevertheless, reaching near Shannon limit performance is only possible with very large interleavers prohibiting an application in speech-transmission. This paper presents simulation results for a short-frame data transmission system based on DS-CDMA (Direct-Sequence Code Division Multiple Access) employing Turbo-Codes. Furthermore, the performance of a turbo-coded modulation scheme is investigated with respect to the typical conditions of DS-CDMA systems. As a result, it is shown that Turbo-Codes are no longer superior to classical convolutional codes if the same decoding costs are assumed. Additionally, simulation results illustrate that coded modulation schemes are not well suited for the application in CDMA systems. Finally, it is proved that an accurate channel estimation is necessary for allowing of high coding gains.
\end{abstract}

\section{INTRODUCTION}

In order to ensure an appropriate performance in digital mobile radio systems, a sophisticated channel coding concept is necessary. In 1993, a novel class of binary parallel concatenated recursive convolutional codes termed Turbo-Codes received great attention because they allow near Shannon limit performance [1]. Furthermore, their combination with bandwidth-efficient modulation schemes termed Turbo-Coded Modulation [2] outperforms the classical trellis coded modulation (TCM) of Ungerboeck [3].

Unfortunately, the amazing results can only be achieved with very large interleaver sizes causing tremendous transmission delays and prohibiting an application in speechtransmission. In [1], a gap of only $0.7 \mathrm{~dB}$ to Shannon's limit remains using a random interleaver of 256 rows and 256 columns $L=65536$ causing a transmission delay of $\tau=13.65 \mathrm{~s}$ at a data rate of $R_{d}=9.6 \mathrm{kbit} / \mathrm{s}$. Nevertheless, the general concept of parallel concatenated codes in combination with an iterative decoding scheme involves a lot of possibilities to improve the performance of modern communication systems. Therefore, it is an important task to investigate whether Turbo-Codes and TurboCoded Modulation are still superior to conventional coding concepts under the constraint of moderate interleaver sizes.

In order to reach this goal, Turbo-Codes and TurboCoded Modulation were investigated for an interleaver size of $L=400$ bits causing a transmission delay of about $\tau=83,3 \mathrm{~ms}$ at a data rate of $R_{d}=9.6 \mathrm{kbit} / \mathrm{s}$. With this restriction, a Direct-Sequence CDMA system is used to compare the performance of Turbo-Codes with that of classical convolutional codes taking into account the computational costs of the specific decoding procedures. Additionally, the influence of an imperfect channel estimation on the coding gain is illustrated.

This paper is organized as follows: Section 2 briefly reviews the general concept of Turbo-Codes and defines some specific parameters concerning generator polynomials and decoding algorithms. Section 3 describes a TurboCoded Modulation scheme [2] and explains details for an optimal mapping strategy. Additionally, it presents a low cost demodulation algorithm supplying soft-output values. Section 4 treats the simulation model and section 5 illustrates the received results.

\section{Turbo-CODES}

\section{A. Turbo-Encoder}

This section briefly describes the concept of turboencoding and-decoding as it is used in this article. The principle structure of the encoder is shown in Figure 1 [1]. Turbo-Codes are systematic codes consisting of at least two parallel concatenated constituent codes $C_{1}$ and $C_{2}$ connected by an interleaver IL. The interleaver approximately ensures statistical independence of the parity bits $\mathbf{c}_{1}(k)$ and $\mathbf{c}_{2}(k)$ at the output of the constituent encoders. Assuming identical constituent codes with rates $R_{c 1}=R_{c 2}=1 / N$, the overall code rate of the unpunctured Turbo-Code is $R_{c}=1 /(2 N-1)$. A larger overall code rate $R_{c}$ can be realized by puncturing $\mathbf{c}_{1}(k)$ and $\mathbf{c}_{2}(k)$ with respect to a matrix $\mathbf{P}$ given in Table I. Each column of $\mathbf{P}$ contains $2(N-1)$ elements associated with the same

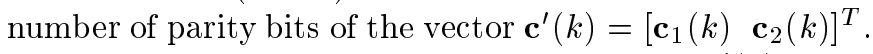
A zero indicates that the corresponding bit of $\mathbf{c}^{\prime}(k)$ is not transmitted. The columns are read periodically one after another so that puncturing is performed with a period identical to the number of columns of $\mathbf{P}$.

In this article, Turbo-Codes with only two constituent codes are considered. As shown in Table I, the overall code rates $R_{c}=1 / 2$ and $R_{c}=2 / 3$ are obtained with constituent codes of rate $R_{c 1}=R_{c 2}=1 / 2$ and appropriate puncturing. The Turbo-Code with $R_{c}=1 / 4$ employs 


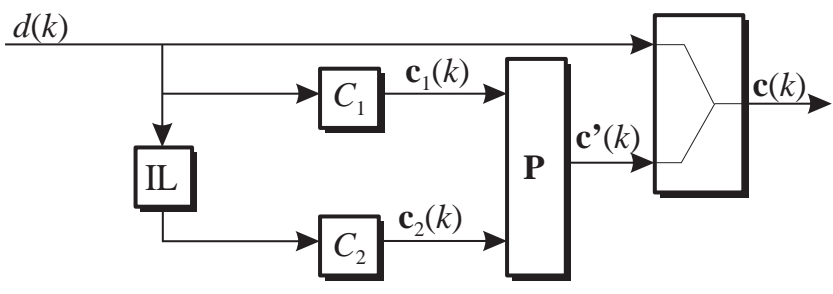

Fig. 1. Structure of the turbo-encoder

constituent codes of rate $R_{c 1}=R_{c 2}=1 / 3$. As derived in [4], systematic and recursive convolutional codes have to be employed in order to take advantage of the interleaver. Furthermore, it is shown in [5] that the effective free distance

$$
d_{\text {eff }}=2+2 \cdot z_{\text {min }}
$$

is a critical parameter to be optimized. In (1) $z_{\min }$ represents the minimum weight of the redundancy bits of a constituent code for an input weight of $w=2$. In order to maximize $d_{e f f}$, constituent codes with primitive feedback polynomials were chosen because they maximize the minimal length of output sequences for an input weight of $w=2$. The generator polynomials and their corresponding puncturing matrices used in this article are given in Table I. The superscript $r$ indicates the recursive polynomial. One aim of this paper is the comparison between Turbo-Codes and convolutional codes assuming the same computational decoding costs. For six decoding iterations a convolutional code with a constraint length of $L_{c}=9$ is required. Here, it has to be mentioned that the data rate is slightly reduced in case of convolutional coding because more tail bits are needed to determine the trellis. Nevertheless, this disadvantage can be compensated by slightly puncturing the convolutional code with negligible performance loss.

TABLE I

GENERATOR POLYNOMIALS FOR DIFFERENT CODE RATES

\begin{tabular}{|c|c|c|c|}
\hline $\begin{array}{l}\text { Overall } \\
\text { code } \\
\text { rate }\end{array}$ & $\begin{array}{c}\text { Convolutional } \\
\text { codes } \\
L_{c}=9\end{array}$ & $\begin{array}{l}\text { Turbo } \\
\text { Codes } \\
L_{c}=5\end{array}$ & $\begin{array}{c}\text { Puncturing } \\
\text { matrix } \\
\mathbf{P}\end{array}$ \\
\hline$R_{c}=\frac{1}{2}$ & $\begin{array}{l}\mathbf{g}_{1}=561_{8} \\
\mathbf{g}_{2}=753_{8}\end{array}$ & $\begin{array}{l}\mathbf{g}_{1}^{r}=23_{8} \\
\mathbf{g}_{2}=35_{8}\end{array}$ & $\left(\begin{array}{ll}1 & 0 \\
0 & 1\end{array}\right)$ \\
\hline$R_{c}=\frac{1}{4}$ & $\begin{aligned} \mathbf{g}_{1} & =463_{8} \\
\mathbf{g}_{2} & =535_{8} \\
\mathbf{g}_{3} & =733_{8} \\
\mathbf{g}_{4} & =745_{8}\end{aligned}$ & $\begin{array}{l}\mathbf{g}_{1}^{r}=23_{8} \\
\mathbf{g}_{2}=35_{8} \\
\mathbf{g}_{3}=25_{8}\end{array}$ & $\left(\begin{array}{ll}1 & 1 \\
1 & 0 \\
1 & 1 \\
0 & 1\end{array}\right)$ \\
\hline$R_{c}=\frac{2}{3}$ & & $\begin{array}{l}\mathbf{g}_{1}^{r}=23_{8} \\
\mathbf{g}_{2}=35_{8}\end{array}$ & $\left(\begin{array}{ll}1 & 0 \\
0 & 0 \\
0 & 0 \\
0 & 1\end{array}\right)$ \\
\hline
\end{tabular}

Concerning the application of Turbo-Codes for speech transmission, the tolerable transmission delay has to be restricted to some $10 \mathrm{~ms}$. For this reason, an interleaver size of $L=400$ bits is chosen, limiting the performance of turbo-codes dramatically. In this work, a a simple block interleaver is used. The performance degradation due to the missing optimization of the interleaver structure can be neglected for the mentioned interleaver size and bit error rate above $P_{b} \geq 10^{-4}[6]$.

\section{B. Turbo-Decoder}

Figure 2 shows the structure of the turbo-decoder. The two decoders $D_{1}$ and $D_{2}$ corresponding to the constituent codes $C_{1}$ and $C_{2}$, respectively, are serially arranged and connected by an interleaver. The extrinsic information $Z_{1}(k)$ of the first decoder $D_{1}$, i.e. the information obtained only from the redundancy bits of $C_{1}$ [1], can be used as a-priori-information for $D_{2}$ and vice versa. As a consequence, after de-interleaving $\left(\mathrm{IL}^{-1}\right)$ the extrinsic information of $D_{2}, Z_{2}(k)$ is passed back to $D_{1}$ and an iterative decoding procedure arises. As a decoding algorithm the Max-Log-MAP-algorithm is applied [7], [8]. Investigations have shown that this is an appropriate compromise between performance, robustness and computational effort [9]. As already mentioned, six decoding iterations are used in the simulations.

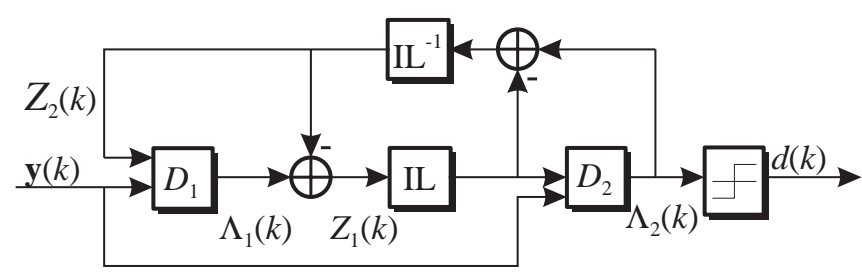

Fig. 2. Structure of the turbo-decoder

\section{Turbo-Coded Modulation}

In many applications, the bandwidth expansion introduced by the coder's redundancy cannot be tolerated. In these cases it is necessary to use a bandwidth-efficient modulation scheme. A first approach combining TurboCodes with a multilevel modulation scheme is presented in [2]. The structure of this approach is depicted in Figure 3. Due to the desired high spectral efficiency only constituent codes of rate $R_{c 1}=R_{c 2}=1 / 2$ are used. Here, $n$ unpunctured code words $\mathbf{c}(k)$ of the turbo-encoder in Figure 1 consisting of 3 bits are arranged in a parallel manner separating the systematic information bits $\mathbf{d}$ and the redundancy bits $\mathbf{c}_{1}$ and $\mathbf{c}_{2}$ of the constituent codes $C_{1}$ and $C_{2}$, respectively. After puncturing the $2 n$ redundancy bits, the resulting $(m-n)$-tuple $\mathbf{c}^{\prime}$ is combined with $n$ information bits to the $m$-tuple $\mathbf{u}(l)$ selecting one of $M=2^{m}$ possible symbols.

Concerning the mapping of $\mathbf{u}(l)$ to a specific symbol $s(l)$, Figure 5 illustrates for the coded 8-PSK that Gray coding offers a gain of more than $2 \mathrm{~dB}$ over Ungerboeck's Set Partitioning [3]. The latter mapping rule is not suited for this approach because it neglects the code structure [9]. It is rather advantageous to perform Gray encoding ensuring that the binary representations of neighboring symbols differ only in 1 bit (s. Figure 4 ). This results in 


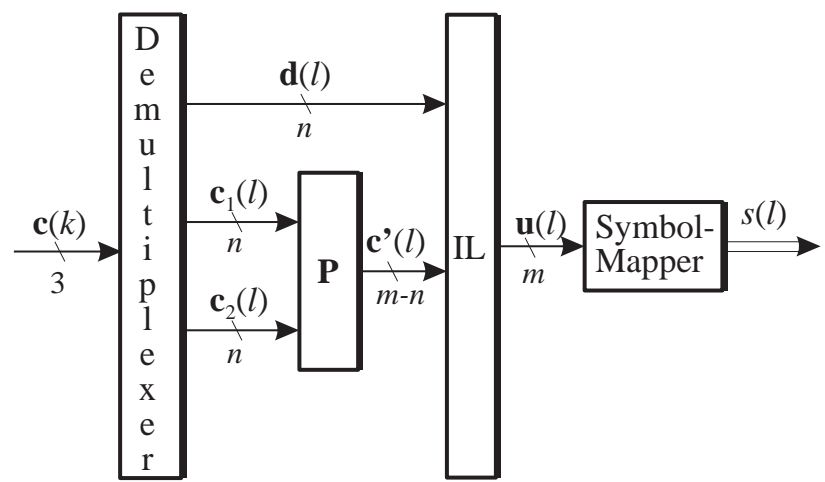

Fig. 3. Combination of turbo-encoder and modulator

a higher average signal-to-noise-ratio after demodulation and consequently in a lower error rate after decoding [9].

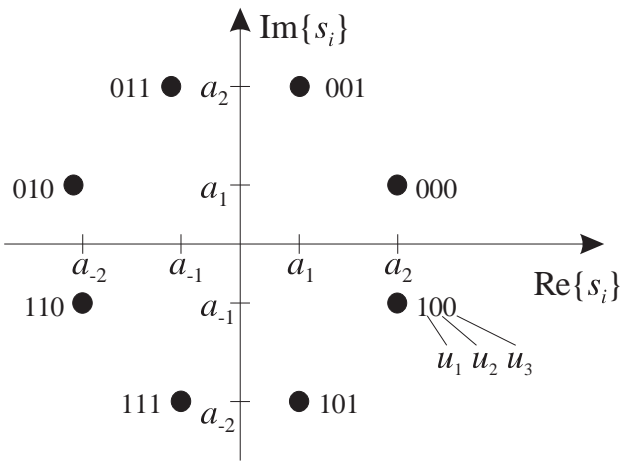

Fig. 4. Signal constellation for 8-PSK and Gray-coding

Another question using Gray-coding is how to map specific bits $c_{i}$ of a code word $\mathbf{c}$ to bits $u_{j}$ of a symbol $s(l)$. Obviously, $u_{1}$ and $u_{2}$ possess a lower error probability than $u_{3}$. Simulation results indicate that the information bits $\mathbf{d}$ and the redundancy bits $\mathbf{c}_{1}$ corresponding to the first encoder $C_{1}$ (if possible) should be assigned to bits $u_{1}$ and $u_{2}$ [9]. Using this strategy, the error rate after the first constituent decoder is lower leading to a lower error rate after the whole decoding procedure. Although the TurboCode's performance itself is independent of the specific mapping, it is advantageous to offer the most reliable bits to the first decoder. This behaviour is caused by the iterative decoding process and it is expected that it does not occur in the case of maximum likelihood decoding of the entire code. In the case of a rate $2 / 3$ coded 8 -PSK the two information bits per symbol should be assigned to $u_{1}$ and $u_{2}$ (labeled as System 1 in Figure 5) leading to a gain of $0.3 \mathrm{~dB}$ over the mapping to $u_{2}$ and $u_{3}$ (labeled as System 2) as depicted in Figure 5.

The separation of encoder and modulator at the transmitter site results in separate demodulation and decoding procedures as shown in Figure 6. Therefore, soft-output demodulation is required to avoid information loss after demodulation. A very simple way supplying reliability information is the exploitation of the symmetric arrangement of symbols in the signal space as depicted in Figure 4 [9]. Then, a pragmatic approach using only the inphase component $x^{\prime}$ and the quadrature component $x^{\prime \prime}$ of the received symbol $x(l)$ leads to the following results for 8-PSK

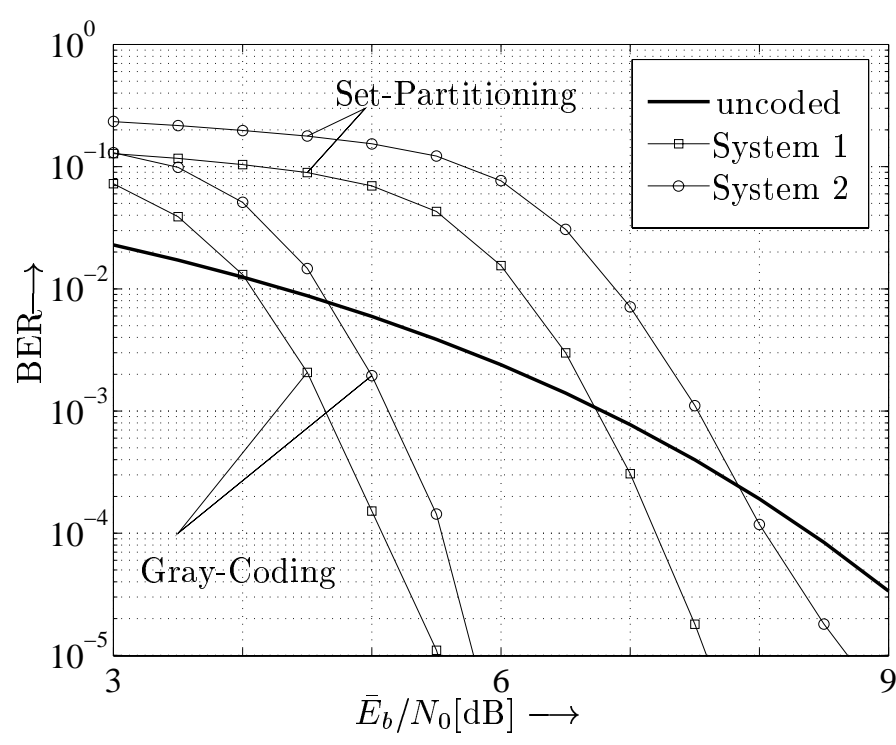

Fig. 5. Bit error rate for 8-PSK and different mapping

modulation [9]:

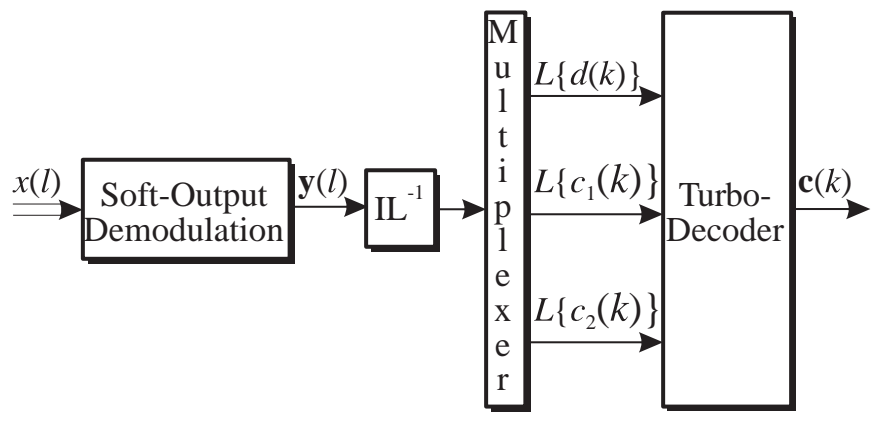

Fig. 6. Combination of demodulator and turbo-decoder

$$
L\left\{u_{j}\right\}=\frac{1}{\sigma^{2}} \cdot \begin{cases}-2 a_{1} \cdot x^{\prime \prime} & j=1 \\ -2 a_{1} \cdot x^{\prime} & j=2 \\ \left(a_{2}-a_{1}\right) \cdot\left(\left|x^{\prime \prime}\right|-\left|x^{\prime}\right|\right) & j=3\end{cases}
$$

where $\sigma^{2}$ describes the noise variance. This expression can be easily extended for higher order modulation. Investigations indicate only slight differences between the optimal MAP-demodulation [9], [2] and pragmatic approach of (2) for 8-PSK modulation. A further simplification was achieved by replacing the estimated noise variance $\sigma^{2}$ by a constant value of $\sigma^{2} \equiv 0.1$ leading only to a minor performance loss. Using this approach, nearly no additional costs are required to supply soft-output information.

Additionally, the interpretation of $L\left\{u_{j}\right\}$ as a $\log$ likelihood-ratio reduces the computational costs of the decoding algorithms. Following the concept in [7], no estimation of the noise variance is necessary.

\section{CDMA Simulation Model}

Figure 7 shows the structure of the simulation model. The data stream $d(k)$ with a rate of $R_{d}=9.6 \mathrm{kbit} / \mathrm{s}$ is first encoded by the channel encoder using conventional convolutional codes (CC) or Turbo-Codes (TC) as described above. After interleaving the code words 


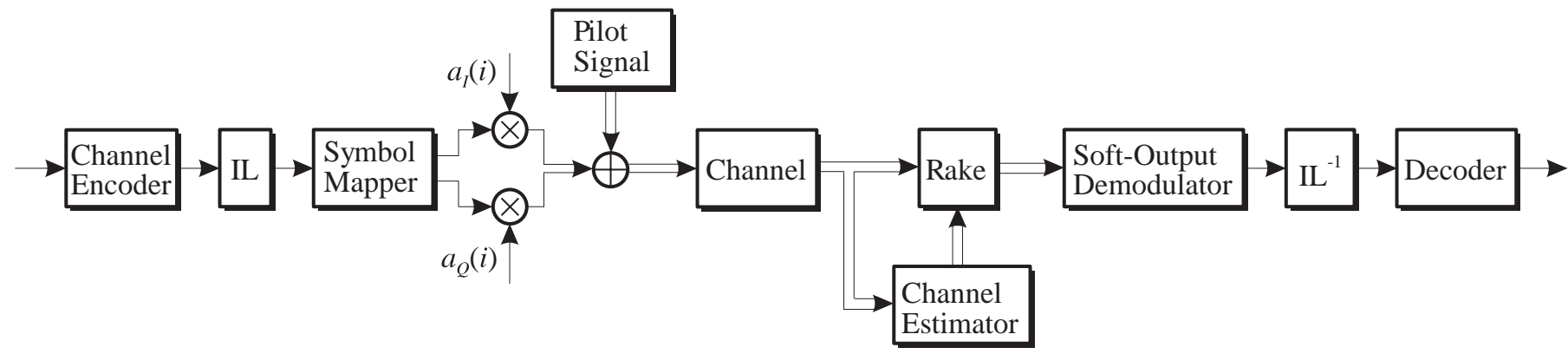

Fig. 7. Structure of CDMA system model

$\mathbf{c}(k)$, they are mapped to PSK-symbols $s(l)$ and DirectSequence Spreading is performed as shown in Table II. In the case of an uncoded transmission the bandwidth is increased by a factor 127 . When channel coding is used, the Direct-Sequence bandwidth expansion is reduced by a factor equal to the code rate. As a consequence, all system configurations are based on approximately the same processing gain of $G_{P} \approx 63$. Direct-Sequence spreading is carried out separately for both inphase and quadrature component. Gold Codes with periods of 31, 63 and 127 are employed.

TABLE II

Parameter CONFiguration

\begin{tabular}{|c|c|c|c|}
\hline Modulation & Interleaver & $\begin{array}{c}\text { DS- } \\
\text { Spreading }\end{array}$ & $\begin{array}{c}\text { Processing } \\
\text { gain }\end{array}$ \\
\hline QPSK & uncoded & 127 & 63,5 \\
\hline $\begin{array}{c}\text { QPSK } \\
R_{c}=1 / 2\end{array}$ & $\begin{array}{c}\text { CC: } 18 \times 45 \\
\text { TC: } 10 \times 80\end{array}$ & 63 & 63 \\
\hline $\begin{array}{c}\text { QPSK } \\
R_{c}=1 / 4\end{array}$ & CC: $36 \times 45$ & 31 & 62 \\
\hline $\begin{array}{c}8-\mathrm{PSK} \\
R_{c}=\frac{2}{3}\end{array}$ & TC: $10 \times 60$ & 31 & 62 \\
\hline
\end{tabular}

The mobile radio channel is modeled as a four-path Rayleigh fading channel with a maximum Doppler frequency shift of $f_{d \max }=200 \mathrm{~Hz}$. Therefore, channel interleaver as shown in Table II are used. The number of rows depends on the code rate and the constraint length of the actual code. The Turbo-Code with its inherent interleaver of 20 rows and 20 columns causes a transmission delay of $83 \mathrm{~ms}$. This delay is not increased by the second external channel interleaver with $n=L_{c} / R_{c}=10$ rows and 80 columns. For the convolutional code with $L_{c}=9$, $n=L_{c} / R_{c}$ rows is a good choice. The number of columns is determined in a way to obtain the same total delay for all configurations. This choice results in a time spacing between two successive bits of $\Delta t=2.34 \mathrm{~ms}$ that suffices for a coherence time of the channel of $t_{c}=2.5 \mathrm{~ms}$.

Furthermore, an unmodulated pilot signal, transmitted with a power being $6 \mathrm{~dB}$ higher than the power of the data channels, delivers the phase reference [10] and is used for the channel estimation [9].

\section{Simulation Results}

This paper pursues two goals: First, Turbo-Codes are to be compared with convolutional codes under the constraint of the same computational decoding effort. Second, a compromise between a high coding gain (low code rate) and long spreading sequences should be worked out. A long spreading sequence possesses better correlation properties and thus yields a better suppression of interference due to multi-path propagation and multi-user interference. This advantage is attached with a high code rate and consequently a small coding gain.

Figure 8 illustrates the bit error rates for convolutional codes, Turbo-Codes and Turbo-Coded Modulation for different code rates. Concerning the convolutional codes, no differences for 1/2-rate and 1/4-rate coding can be observed. The Turbo-Code with rate $1 / 4$ performs as well as the latter ones, whereas the rate $1 / 2$-Turbo-Code has a lower performance. The turbo-coded 8-PSK loses up to $1 \mathrm{~dB}$ over the other configurations caused by the higher signal density in combination with a higher code rate. These disadvantages cannot be compensated by better correlation properties of long spreading sequences [9].

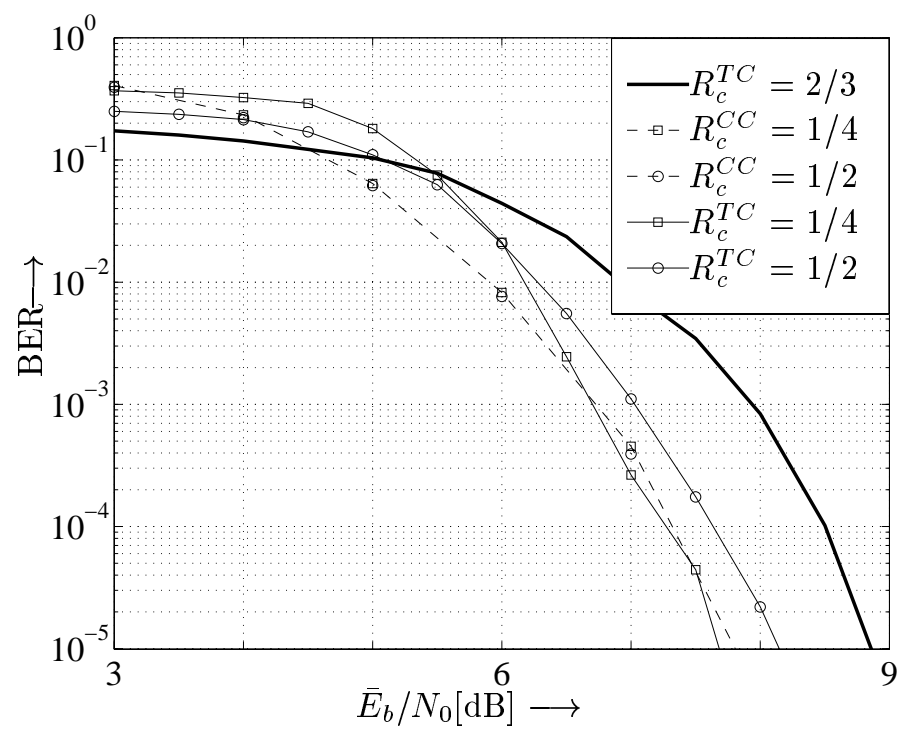

Fig. 8. Bit error rate for different coding scenarios

Surprisingly, it can be recognized that there occurs only 
a small difference between code rates of $1 / 4$ and $1 / 2$. This observation can be explained with the inaccurate channel estimation. Although the pilot signal is transmitted with a higher power in comparison with the data signals, the signal-to-noise-ratio is still too low. In order to reduce the effect of the background noise ten estimated coefficients were averaged leading to more accurate estimates of the channel coefficients [9].

Figure 9 shows the bit error rates for perfectly known channel coefficients, conventionally estimated coefficients and the approach by averaging 10 estimated coefficients. Obviously, the accuracy of the channel estimation determines strongly the performance of the channel coding. Averaging over ten estimated values leads to a gain of $3 \mathrm{~dB}$ over the estimation without averaging, there remains only a loss of $1 \mathrm{~dB}$ over perfectly known coefficients.

Additionally, a code rate of $1 / 4$ with a spreading sequence of length 31 now outperforms the code with rate $1 / 2$ and a spreading sequence with period 63 by $1 \mathrm{~dB}$. These results lead to the conclusion that the advantages of powerful channel codes with low code rate can only be exploited with an accurate estimation of the channel state. The difference between perfectly known coefficients and conventional estimation for the coded 8-PSK modulation is only $1 \mathrm{~dB}$, so additional averaging will not result in large gains and is therefore not considered here.

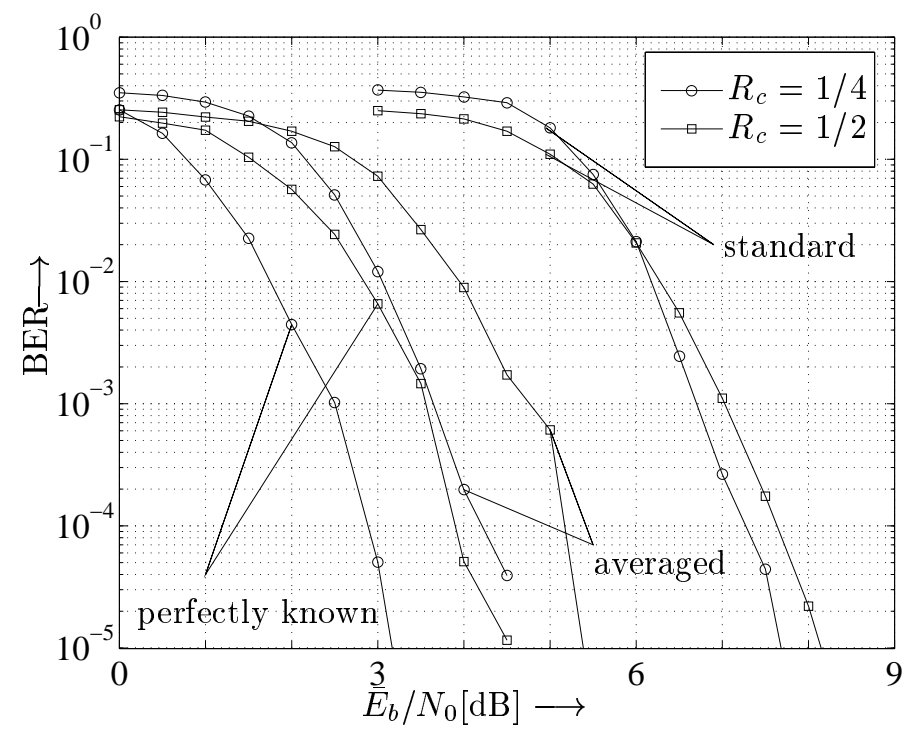

Fig. 9. Bit error rate for perfectly known and estimated channel coefficients and Turbo-Codes

Finally, the performance is checked under the consideration of ten additional users causing mulit-user interference. As can be seen in Figure 10, the difference between rate $1 / 4$ and rate $1 / 2$ coding is reduced to only $0.5 \mathrm{~dB}$. The longer Gold-sequence of length 63 (s. Table II) ensures a better estimation of the channel and a better suppression of additional interfering user signals. Concerning the coded 8-PSK the unsatisfying performance is obvious.

\section{Conclusion}

It is shown that Turbo-Codes are no longer superior to convolutional codes if the interleaver size is restricted

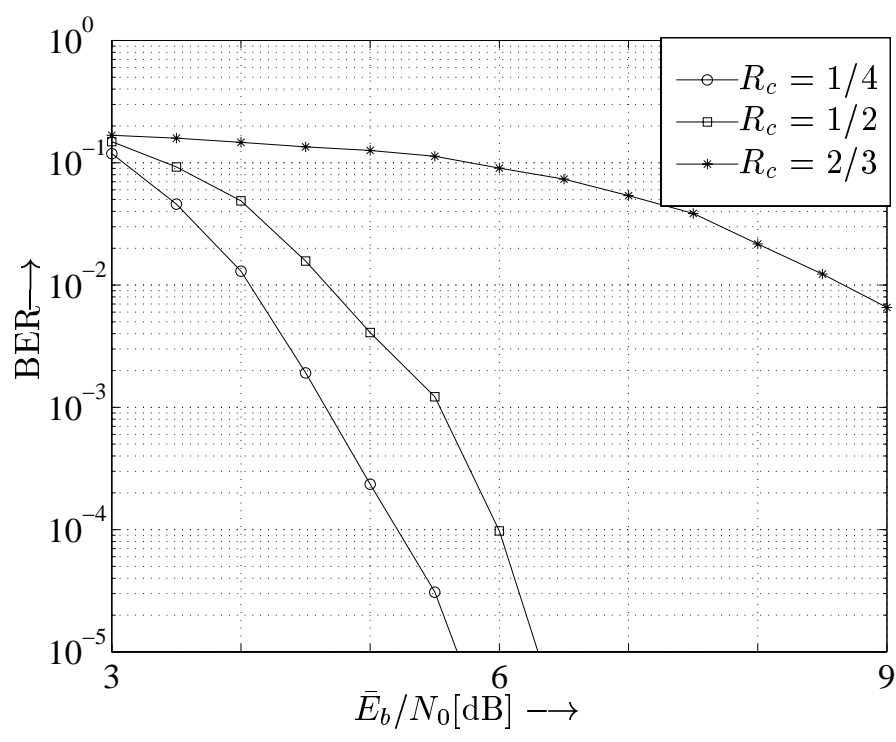

Fig. 10. Bit error rate for additional users and Turbo-Codes

to some hundred bits and the decoding effort is approximately the same. Furthermore, a coded modulation scheme is not attractive in a CDMA-system. It is rather advantageous to decrease the code rate and to shorten the spreading sequence. In order to take advantage of the more powerful code, an accurate channel estimation is necessary. This can be realized by averaging 10 estimated channel coefficients leading to a performance gain of up to $3 \mathrm{~dB}$.

\section{REFERENCES}

[1] C. Berrou, A. Glavieux, and P. Thitimajshima: "Near shannon limit error-correcting coding and decoding: Turbo-codes (1)", In Proc. IEEE International Conference on Communications (ICC'93), pages 1064-1070, Geneva, 1993.

[2] S. Le Goff, A. Glavieux, and C. Berrou: "Turbo-codes and high spectral efficiency modulation", In Proc. IEEE International Conference on Communications (ICC'94), pages 645-649, New Orleans, 1994.

[3] G. Ungerboeck: "Channel coding with multilevel phase signaling", IEEE Transactions on Information Theory, Vol. IT-25, pages 55-67, 1982.

[4] S. Benedetto and G. Montorsi: "Unveiling turbo-codes: Some results on parallel concatenated coding schemes", IEEE Transactions on Information Theory, Vol. 42, No. 2, pages 409-428, March 1996.

[5] S. Benedetto and G. Montorsi: "Design of parallel concatenated convolutional codes", IEEE Transactions on Communications, Vol. 44, No. 5, pages 591-600, May 1996.

[6] J. Hagenauer, P. Robertson, and L. Papke: "Iterative (turbo)decoding of systematic convolutional codes with the map and sova algorithms", In Proc. ITG Fachtagung'Codierung', Munich (Germany), pages 21-29, October 1994.

[7] P. Robertson, E. Villebrun, and P. Hoeher: "A comparison of optimal and sub-optimal map decoding algorithms operating in the log domain", In International Conference on Communications (ICC'95), June 1995.

[8] P. Jung: "Comparison of turbo-code decoders applied to short frame transmission systems", IEEE Journal on Selected Areas in Communications, Vol. 14, No. 3, pages 530-537, April 1996.

[9] V. Kühn: Turbo-Codes und turbo-codierte Modulation in Codemultiplex-Mobilfunksystemen, $\mathrm{PhD}$ thesis, University of Paderborn, 1998.

[10] V.Kühn and M. Meyer: "Correlative channel estimation in DSCDMA systems", In Third Annual WIRELESS Symposium, pages 279-284. Penton Publishing, 1995. 